\title{
Flux and source-sink relationship of heavy metals and arsenic in the Bohai Sea, China ${ }^{\text {25 }}$
}

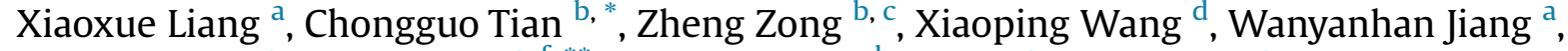 \\ Yingjun Chen ${ }^{\mathrm{e}}$, Jianmin Ma ${ }^{\mathrm{a}, \mathrm{f},{ }^{* *}}$, Yongming Luo ${ }^{\mathrm{b}}$, Jun $\mathrm{Li}^{\mathrm{c}}{ }^{\mathrm{c}}$, Gan Zhang ${ }^{\mathrm{c}}$ \\ ${ }^{a}$ Key Laboratory for Environmental Pollution Prediction and Control, College of Earth and Environmental Sciences, Lanzhou University, Lanzhou, 730000, \\ China \\ ${ }^{\mathrm{b}}$ Key Laboratory of Coastal Environmental Processes and Ecological Remediation, Yantai Institute of Coastal Zone Research, Chinese Academy of Sciences, \\ Yantai, 264003, China \\ c State Key Laboratory of Organic Geochemistry, Guangzhou Institute of Geochemistry, Chinese Academy of Sciences, Guangzhou, 510640, China \\ ' Ludong University, Yantai, 264025, China \\ e Key Laboratory of Cities' Mitigation and Adaptation to Climate Change in Shanghai (CMA), College of Environmental Science and Engineering, Tongji \\ University, Shanghai, 200092, China \\ ${ }^{\mathrm{f}}$ Laboratory for Earth Surface Processes, College of Urban and Environmental Sciences, Peking University, Beijing, 100871, China
}

\section{A R T I C L E I N F O}

\section{Article history:}

Received 12 June 2018

Received in revised form

3 August 2018

Accepted 3 August 2018

Available online 7 August 2018

\section{Keywords:}

Source-sink relationship

Heavy metals

Arsenic

Water exchange

Bohai sea

North Yellow sea

\begin{abstract}
A B S T R A C T
This study conducted a field campaign to collect atmospheric deposition samples of heavy metals and arsenic, a metalloid element with typical chemical-physical characteristics (HMA), from 12 sampling sites and water samples from 37 rivers across the Bohai Sea (BS) and North Yellow Sea (NYS) in China. The HMA budgets in the BS and NYS were quantified by a budget model, which was developed based on the HMA inputs from atmospheric deposition and riverine discharge, sequestration to sediment, and interexchange among the BS's four subareas and the NYS. Statistical analyses of 76 deposition samples and 109 water concentration samples showed that atmospheric deposition was a main pathway of $\mathrm{Pb}$ entering the BS and NYS, whereas riverine discharge dominated the input of $\mathrm{Cr}, \mathrm{Cu}, \mathrm{Zn}, \mathrm{Cd}$, and As into the marine environment. Modeled results showed that the fractions of HMA in the water bodies compared with their total burdens were $86.6 \pm 4.55 \%$ in the Liaodong Bay, $60.5 \pm 10.5 \%$ in the Bohai Bay, $20.9 \pm 9.05 \%$ in the Laizhou Bay, $95.1 \pm 2.06 \%$ in the Central BS, and $94.3 \pm 1.93 \%$ in the NYS. The lowest fraction of HMA in the Laizhou Bay was attributed to high sedimentation rates and higher suspended particulate matter concentrations due to inputs from the Yellow River. The modeled 1-, 10- and 100- year mass budgets indicated that the Liaodong Bay in the north of the BS was a sink of HMA, the Bohai Bay and Laizhou Bay in the west and south of the BS acted as sources, and the Central BS and NYS were a transition area for most HMA.
\end{abstract}

๑ 2018 Elsevier Ltd. All rights reserved.

\section{Introduction}

The Bohai Sea (BS) watershed is one of the most populated and industrialized regions in China. With rapid economic development and urbanization over the past few decades around the BS, the marine ecosystem has been heavily contaminated by the input and discharge of toxic chemicals through the atmosphere, runoff, and surrounding rivers. Considerable attention has been paid to the environmental pollution of the BS due to the significance of the BS Rim to China (Duan and Li, 2017; Gao et al., 2014). In the coastal area of the BS, which is known as the BS Economic Rim (BSER), there are four key national economic zones, including the Tianjin Binhai New Area, Liaoning Coastal Economic Zone, Yellow River Delta Eco-economic Zone, and Blue Economic Zone of Shandong

\footnotetext{
This paper has been recommended for acceptance by Dr. Joerg Rinklebe.

* Corresponding author.

** Corresponding author. Ludong University, China.

E-mail addresses: cgtian@yic.ac.cn (C. Tian),jmma@pku.edu.cn, jianminma@lzu.edu.cn (J. Ma).
} 
Peninsula, as of 2000 (Liu et al., 2014). The establishment of these economic zones has led to rapid population aggregation and intensive expansion of urbanization and industrialization in the BSER (Wu et al., 2016), thereby putting the BSER at risk to environmental stress (SOA(State-Oceanic-Administration-of-China) 2017). A number of surveys demonstrated that the estuary and bay ecosystems in the BS have been in sub-healthy and unhealthy states in recent years (Gao et al., 2014) and heavy metals and arsenic (HMA) were some of the most important pollutants ( $\mathrm{Hu}$ et al., 2017, SOA(State-Oceanic-Administration-of-China) 2017, Zhang and Gao 2015).

Identification of the flux and source-sink relationship of HMA in the BS is vital in order to design effective programs and strategies to regulate and restore its ecosystem (Fang ets al. 2015). To improve the BS's environmental condition, the Chinese government made great efforts and launched national programs, such as the 'Bohai Blue Sea Action Plan' in 2001 (Gao et al., 2014). However, the programs have not achieved sufficient success (Gao et al., 2014). The flux and source-sink relationship of pollutant in the BS were also not established. Nevertheless, many sampling campaigns under the umbrella of this program and other national programs were launched to assess the BS's environmental condition. These campaigns measured various chemical markers, including nutrients, organic compounds, and toxic heavy metals in the water column, biota, and sediments of the BS (Gao et al., 2014; Meng et al., 2017; Zhang et al., 2009). Of the many pollutants identified, HMA have been identified as the most prevalent pollutants leading to the degeneration of the BS ecosystem (SOA(State-OceanicAdministration-of-China) 2017). HMA are often used as tracers in the assessment of source-sink relationship of pollutant because they are the most persistent and do not decompose in the environment (Liu et al., 2017; Pan and Wang, 2012).

The input and output fluxes of HMA are crucial information for building their source-sink relationship in the BS. Usually, atmospheric deposition and river runoff are considered the primary input pathways of HMA entering the BS (Duan and Li, 2017). Their burial in sediments can be treated as the dominant output pathway (Fang et al., 2015) and their exchange between the BS and the North Yellow Sea (NYS) can be viewed as the dominant output or input pathway (Fang et al., 2015; Wang et al., 2007). However, the knowledge about the HMA source-sink relationship gained from previous studies was disproportionate. Data on the HMA environmental loadings in the BS sediments are the most abundant. For instance, a recent review article summarized more than 3000 HMA concentration records in the BS sediments (Duan and Li, 2017). The second amplest data are the HMA concentrations in the water bodies of the BS and its estuaries (Wang and Wang, 2007; Xu et al., 2013a). Regarding these estuaries, more concern has been paid to large rivers, such as the Yellow River (Bi et al., 2014; Tang et al., 2010; Wang et al., 2016) and Liao River (Yang et al., 2015). While there are more than 40 rivers entering the BS (Gao et al., 2014), a systematic and comprehensive HMA survey of estuaries has not been conducted. In particular, there is a large knowledge gap regarding HMA atmospheric deposition to the BS due to scarce measurements.

This study conducted a sampling campaign to collect atmospheric deposition samples and riverine water samples across the BS and NYS to fill knowledge and data gaps of HMA. A model was developed to quantify HMA source-sink relationship, which took into account the measured land-source input, burial into sediments, and transport by water exchange among the BS's four subareas and the NYS. The major objectives of the present study were (1) to examine the spatiotemporal patterns of atmospheric deposition rates and river concentrations of HMA, (2) to assess the HMA fluxes via atmospheric deposition and river discharge and their respective contributions to the total budget, and (3) to establish source-sink relationship of HMA in the BS and NYS.

\section{Materials and methods}

\subsection{Sampling site and sample collection}

The BS is a semi-enclosed and shallow marginal sea of the northwestern Pacific Ocean on the northern coast of China. Administratively, the BS is adjacent to the Liaoning, Hebei and Shandong Provinces, as well as the Tianjin Municipality. The BS can be geographically divided into four parts, including the Liaodong Bay in the north, Bohai Bay in the west, Laizhou Bay in the south, and the Central BS. The BS is connected to the NYS on its east edge through the Bohai Strait, as shown in Fig. 1. The NYS is separated from the South Yellow Sea to the south by a boundary from Chengshantou at the east edge of the Shandong Peninsula to Changsangot on the Korean Peninsula (Li et al., 2016). The NYS and Bohai Strait are the major channels of matter exchange between the BS and open seas (Liu et al., 2009).

The sampling area in the present study covered the BS and NYS in China. Atmospheric bulk deposition samples (mixture of dry and wet deposition) were collected seasonally from summer 2014 to winter 2015 at 12 sampling sites, as shown in Fig. 1 . A total of 76 bulk deposition samples were collected during the sampling period. The details are described in Text S1 and Table S1 of Supporting Information (SI). Water samples were collected from 37 major rivers around the BS and NYS in 2015. The total water volume of the 37 rivers accounts for more than $98 \%$ of the total river discharge into the BS and NYS (Wang et al., 2015). The samples in normal, rainy, and dry seasons were collected from late May to early June, from late August to early September, and from late November to early December 2015, respectively. Three or five water samples collected along one transect were mixed to form a single sample, which was then immediately filtered through an acidtreated microporous filter $(0.45 \mathrm{~mm}$ mesh $)$ into pre-cleaned highdensity polyethylene containers in the field. The filtered samples were acidified to $\mathrm{pH}<2$ with $\mathrm{HNO}_{3}$ and then delivered to our laboratory, where they were kept at $4{ }^{\circ} \mathrm{C}$ before further analyses. A total of 109 water samples were collected. The detailed information is presented in Text S1 and Table S2 of SI.

\subsection{Sample analysis}

Bulk deposition samples were presented as water solutions, and the same method was used to analyze HMA in deposition samples and river water samples (Sharma et al., 2008). Before analysis, some of the insects and large biological debris were removed from samples. Original water samples were filtered using a sand core filter with a polyether sulfone (PES) membrane filter $(0.2 \mu \mathrm{m})$. The filtrate was collected in a glass bottle and was directly measured for soluble HMA using Inductively coupled plasma mass spectrometry (ICP-MS, PerkinElmer ELAN DRC II). PES membranes were subjected to 24-h equilibration at $25^{\circ} \mathrm{C} \pm 1{ }^{\circ} \mathrm{C}$ temperature and $50 \% \pm 2 \%$ relative humidity before and after use. Loaded filters were put into a Teflon digestion tank and $5 \mathrm{~mL}$ of high-purity $\mathrm{HNO}_{3}$ were added for microwave digestion. The supernatant of the digested solution was used to measure the particle fraction of HMA using ICP-MS. Six HMA, including Lead (Pb), chromium ( $\mathrm{Cr})$, copper $(\mathrm{Cu})$, zinc $(\mathrm{Zn})$, cadmium (Cd), and arsenic (As) in the field samples were determined in the field samples. Atmospheric deposition rates $(\mu \mathrm{g}$ $\mathrm{m}^{-2}$ day $^{-1}$ ) were calculated using the measured total amount of HMA divided by the area of the sampler mouth and the sampling times.

Duplicate blanks and standard reference materials (Chinese 


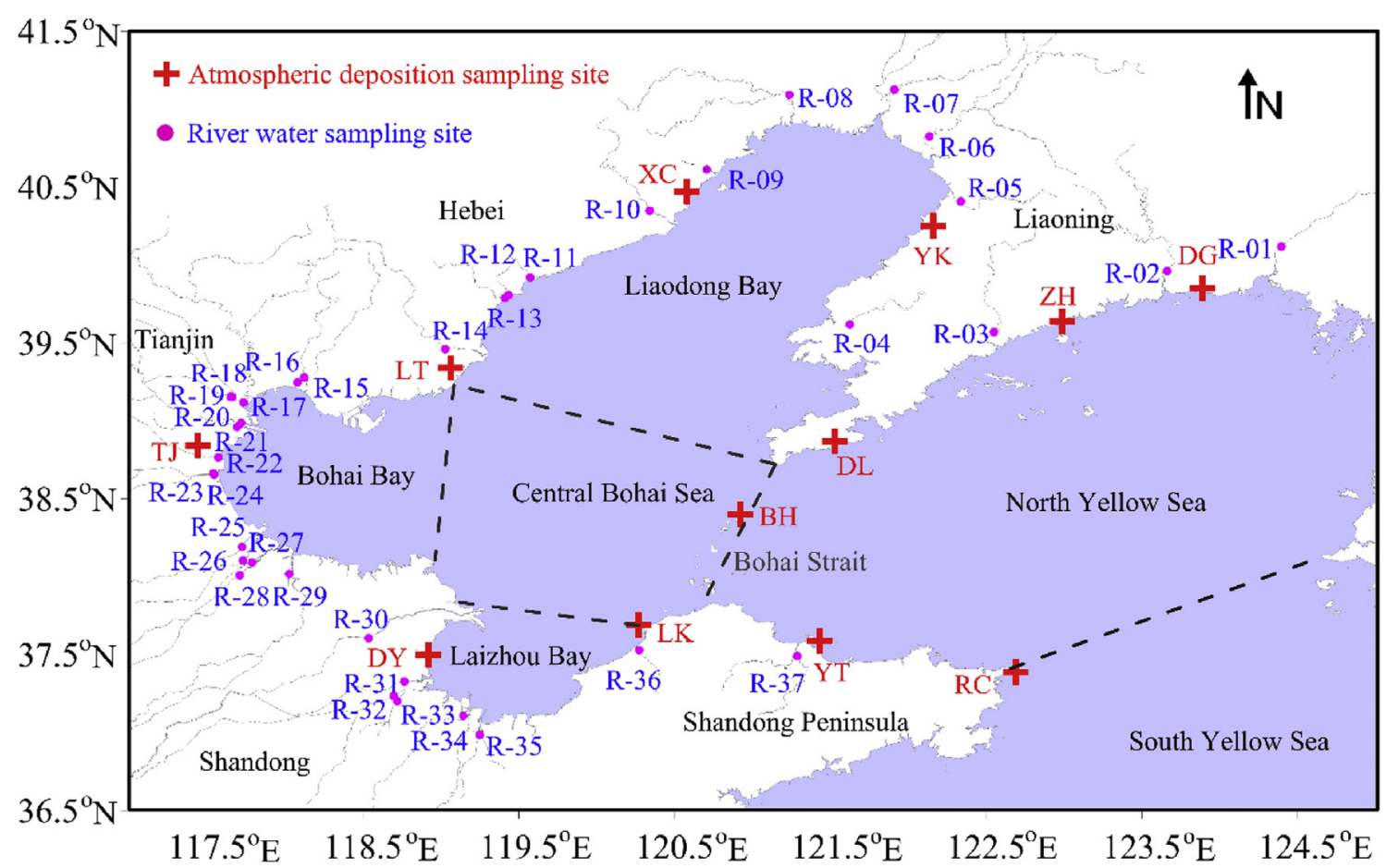

Fig. 1. The sampling sites of atmospheric deposition and riverine water across the BS and NYS and the sea subareas.

National Standards GB/T 23942-2009) were also analyzed to ensure the quality of the measurements. Blank samples were randomly inserted into the analysis process. The blank concentration was less than $\pm 5 \%$ of the mean of the measured HMA concentrations, thereby confirming that the solvent extraction process and the vessel containing the sample did not cause contamination.

\subsection{Flux assessment of atmospheric deposition and river runoff}

Seasonal atmospheric deposition fluxes of HMA in a specific sea area $\left(F_{A}\right.$ in tons) were calculated by the deposition rates at several specified sampling sites, as follows:

$F_{A}=10^{-12} \times \frac{1}{K} \times \sum_{i=1}^{K} D_{A}(i) \times A_{r} \times D_{n}$

where $D_{A}(i)\left(\mu \mathrm{g} \mathrm{m}^{-2}\right.$ day $\left.^{-1}\right)$ is the seasonal deposition rate at the $i t h$ sampling site, $A r$ is the sea area, $D_{n}$ is the day number of each season (spring, summer, autumn, and winter), $K$ is the number of sampling sites, and the value of $10^{-12}$ is the conversion factor from $\mu \mathrm{g}$ to tons. The present study calculated HMA deposition fluxes to the BS's four subareas (including the Liaodong Bay, Bohai Bay, Laizhou Bay, and Central BS) and the NYS. The deposition fluxes of HMA to each subarea were calculated using the deposition rates measured at the sampling sites proximate to the subarea. Specifically, the deposition rates monitored at the sampling sites of DL, YK, XC, and LT (see Fig. 1) were used to evaluate seasonal fluxes to the Liaodong Bay. Similarly, the deposition fluxes to the Bohai Bay were assessed using the measured data at the sampling sites of LT, TJ, and DY; those to the Laizhou Bay were assessed using the data at the DY and LK; those to the Central BS were assessed using data at the DL, LT, $\mathrm{DY}, \mathrm{LK}$, and $\mathrm{BH}$; and those to the NYS assessed using the data the DG, ZH, DL, BH, YT, and RC. The areas of these subareas are listed in Table S3 of SI. The annual deposition fluxes were summed by seasonal deposition fluxes in the four seasons.
Similarly, seasonal HMA discharge fluxes ( $F_{R}$ in tons) for each sea area were calculated by the following equation:

$F_{R}=10^{-9} \times \frac{1}{N} \times \sum_{j=1}^{N}\left[C_{s o}(j)+C_{p a}(j)\right] \times V_{r}$

where $C_{s o}(j)$ and $C_{p a}(j)\left(\mu \mathrm{g} / \mathrm{L}\right.$ or $\left.\mathrm{mg} / \mathrm{m}^{3}\right)$ are soluble and particulate HMA concentrations in the $j$ th river, respectively, $V_{r}$ is the runoff amount of each river $\left(\mathrm{m}^{3}\right), N$ is the number of rivers, and $10^{-9}$ is the conversion factor from $\mathrm{mg}$ to tons. The annual runoff of each river is listed in Table S2 of SI. The fractions of annual runoff in rainy, normal, and dry season were estimated as $0.7,0.2$, and 0.1 , respectively, according to the rainfall fractions listed in Table S2 of SI. The 37 rivers were classified according to their estuaries located in the Liaodong Bay, Bohai Bay, Laizhou Bay, and NYS as listed in Table S2 of SI.

\subsection{Calculation of source-sink relationship}

For a sea area, the net HMA budget includes inputs and outputs. The input pathways are comprised of atmospheric deposition $\left(F_{A}\right.$, ton $\left.\mathrm{yr}^{-1}\right)$, riverine discharge $\left(F_{R}\right.$, ton $\left.\mathrm{yr}^{-1}\right)$, and inflow from other sea areas $\left(F_{I}\right.$, ton $\left.\mathrm{yr}^{-1}\right)$. The output pathways consist of sequestration to the bottom sediments $\left(F_{S}\right.$, ton $\left.\mathrm{yr}^{-1}\right)$ and export to other sea areas $\left(F_{E}\right.$, ton $\left.\mathrm{yr}^{-1}\right)$. The difference between the input and output is the burden in the seawater $\left(F_{W}\right.$, ton $\left.\mathrm{yr}^{-1}\right)$ (Fang et al., 2015; Wang et al., 2007). The net budget can be defined according to the following equation:

$F_{A}+F_{R}+F_{I}=F_{S}+F_{E}+F_{W}$

where $F_{A}$ and $F_{R}$ are calculated using the methods described in Section 2.3. $F_{I}$ and $F_{R}$ are calculated using Eq. (4) and Eq. (5), respectively. 
$F_{I}=10^{-9} \times C_{I} \times Q_{I}$

$F_{E}=10^{-9} \times C_{E} \times Q_{E}$

where $C_{I}\left(\mu \mathrm{g} \mathrm{L}^{-1}\right)$ and $C_{E}\left(\mu \mathrm{g} \mathrm{L}^{-1}\right)$ are HMA concentrations in external and internal water bodies, respectively, for a target sea area. $Q_{I}\left(\mathrm{~m}^{3}\right)$ and $Q_{E}\left(\mathrm{~m}^{3}\right)$ are water inflow flux and water export flux from a target sea area, respectively. The value of $10^{-9}$ is the unit conversion factor. To assess the HMA source-sink relationship of the BS's four subareas and the NYS together, water exchange fluxes among these sea areas were described using a transportation matrix (Su et al., 2011). A particular element $m_{p q}$ in the matrix indicates the percentage of water volume in sea area $p$ from sea area $q$ via 1-year water exchange. The residual fraction of water volume $\left(m_{i i}\right)$ for sea area $i$ after 1 year of water exchange was calculated using the following equation:

$m_{i i}=\exp \left(-\frac{\ln 2}{H_{i}}\right)$

where $H_{i}$ is a half cycle of water exchange in sea area $i$ as listed in Table S4 of SI. The inflow fraction (1- $m_{i i}$ ) was distributed linearly according to the contribution ratios in the transportation matrix under the half-cycle water exchange (see Table S5 of SI). The fractions of water exchange among the BS's four subareas and the NYS were estimated based on the mass balance of water volume (Sun and Tao, 2006). The calculated transportation matrix of the 1-year water exchange is listed in Table S6 of SI. Detailed description and verification of the matrix are described in Text S2 of SI.

When HMA enter the marine environment, they can be adsorbed onto suspended particulate matter (SPM) in adjacent shelf regions, and can be scavenged from the waterbody by sedimentation. Thus, HMA concentrations in SPM were extracted, and the sink fluxes $\left(F_{s}\right.$, ton $\left.\mathrm{yr}^{-1}\right)$ were calculated according to the following equation:

$F_{S}=\frac{10^{-11} \times K_{p} \times \rho \times \omega \times(1-\phi) \times A \times C_{T}}{\left(1+10^{-6} \times S P M \times K_{p}\right)}$

where $K_{p}$ is the partitioning coefficient $\left(\mathrm{L} \mathrm{kg}^{-1} \mathrm{dw}\right)$, which indicates the integrated effects of adsorption and desorption of HMA onto SPM; $\rho$ is the dry density of the sediment samples $\left(\mathrm{g} \mathrm{dw} \mathrm{cm}^{-3}\right) ; \omega$ is the sedimentation rate $\left(\mathrm{cm} \mathrm{yr}^{-1}\right) ; \phi$ is the sediment porosity (dimensionless); $A$ is the area of a given region $\left(\mathrm{m}^{2}\right) ; C_{T}$ is the total HMA concentrations in SPM and water column; SPM is the concentrations of SPM $\left(\mathrm{mg} \mathrm{L}^{-1}\right)$; and $10^{-11}$ is the unit conversion factor. The detailed calculation process and specific values used in the equation are presented in Text S2 and Table S3 of SI.

The HMA source-sink relationship model was solved using Code::Blocks 16.01 software. The net budgets via 100-year water exchange were simulated using the model. The Monte Carlo method was used to assess the net budget variability in the 1-year model scenario. In the Monte Carlo simulation, the model was run repeatedly 1000 times with random selections of $F_{A}, F_{R}, \rho, \omega, \phi$, and $S P M$ according to their means and standard deviations.

\section{Results and discussion}

\subsection{Atmospheric deposition rates}

The mean bulk deposition rates of HMA (including $\mathrm{Pb}, \mathrm{Cr}, \mathrm{Cu}, \mathrm{Zn}$, As, and (d) from summer 2014 to winter 2015 at the 12 sampling sites along the BS and NYS are summarized in Table 1. Only the total deposition rates were reported here because the soluble fractions accounted for more than $99.99 \%$ of their total masses. The mean deposition rates were $183 \pm 430 \mu \mathrm{g} \mathrm{m}^{-2}$ day $^{-1}$ for $\mathrm{Zn}$, $20.5 \pm 20.5 \mu \mathrm{g} \mathrm{m}^{-2} \mathrm{day}^{-1}$ for $\mathrm{Pb}, 4.87 \pm 3.37 \mu \mathrm{g} \mathrm{m}^{-2} \mathrm{day}^{-1}$ for $\mathrm{Cu}$, $1.81 \pm 0.77 \mu \mathrm{g} \mathrm{m}^{-2} \mathrm{day}^{-1}$ for $\mathrm{Cr}, 1.04 \pm 0.47 \mu \mathrm{g} \mathrm{m}^{-2} \mathrm{day}^{-1}$ for As, and $0.19 \pm 0.11 \mu \mathrm{g} \mathrm{m}^{-2} \mathrm{day}^{-1}$ for Cd. Multiple statistical treatments showed insignificant spatiotemporal variation in deposition rates, thereby indicating the pervasiveness and continuity of HMA source emissions over the BS and NYS regions. More details are given in Text S3 and Table S7-S11 of SI.

The deposition rates were used to assess HMA deposition fluxes to the BS and NYS, which is discussed later in this study. Here, a major concern is whether the actual deposition fluxes were overestimated using our data because most sampling sites were proximate to the coastal area rather than to the seawaters (see Fig. 1). This might question the representativeness of the estimated deposition fluxes of HMA to the BS and NYS. To prove the representativeness, we conducted extensive comparisons between measured fluxes in this study and fluxes measured in other areas of China. Results showed that the atmospheric deposition rates around the BS and NYS were lower than those measured in the Pearl River Delta (Wong et al., 2003), North China (Pan and Wang, 2015), Beijing (Guo et al., 2017), and agro-ecosystems in China (Zhang et al., 2017b). However, these deposition rates were comparable with the deposition levels monitored in the Jiaozhou Bay and in several Chinese terrestrial ecosystems (Xing et al., 2017; Zhu et al., 2016). The detailed results of the comparisons are presented in Table S12 of SI. Overall, the results manifested that the measured data in the present study could adequately reflect the deposition levels on a regional scale.

As shown in Fig. 1, a monitoring site (BH) was set on an island in the transect of the Bohai Strait at which the monitored HMA levels could present their means over the sea (see Fig. 1 and Text S1 of SI). The highest deposition rates of $\mathrm{Cu}$, As and $\mathrm{Cd}$, the second highest deposition rates of $\mathrm{Zn}$ and $\mathrm{Pb}$, and the third highest deposition rate of $\mathrm{Cr}$ were identified at this sampling site (see Table 1). The deposition rates in the BS and NYS were not significantly lower than those in the adjacent coastal land. To verify the high deposition levels over the sea surfaces, we further compared the concentrations of HMA bonded in $\mathrm{PM}_{2.5}$ collected from BH, LK, DY, and Tuoji Island in previous studies, and from cities near the BS and NYS (see Table S13 of SI). The HMA concentrations bonded in $\mathrm{PM}_{2.5}$ during August 2014 and September 2015 at BH were higher than those measured from January to February 2014 at Jimu Island in Longkou (same as the LK site in this study), and were comparable with those sampled during May and July 2013 at the Yellow River Delta in Dongying (same as the DY site in this study) (Zong et al., 2017; Zong et al. 2018; Zong et al. 2016). The levels of the most predominant HMA at the BH site were also comparable with those reported in cities near the BS and NYS, such as Dalian, Jinzhou, Fushun, and Anshan (Duan and Tan, 2013). In addition, an atmospheric transport and deposition simulation of Pb was performed (Xu et al., 2013b) and the modeled deposition rates were correlated with the monitored ones (see Figure S1 of SI). The results showed a good correlation when $\mathrm{Pb}$ deposition rate at $\mathrm{BH}$ was left out, suggesting that there was an important source of Pb on the sea (Zhang et al., 2018). Detailed description is illustrated in Text S3 of SI. In general, the comprehensive analyses indicated that the deposition rates in the present study were typical across the BS and NYS.

\subsection{River concentrations}

The soluble and particulate concentrations of HMA in water averaged over 37 rivers around the BS and NYS are summarized in Fig. 2. The mean and standard deviations are listed in Table S14 of 
Table 1

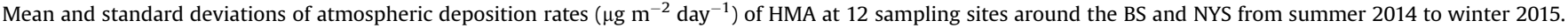

\begin{tabular}{|c|c|c|c|c|c|c|}
\hline Site & $\mathrm{Pb}$ & $\mathrm{Cr}$ & $\mathrm{Cu}$ & $\mathrm{Zn}$ & As & $\mathrm{Cd}$ \\
\hline DG & $7.91 \pm 6.62$ & $3.42 \pm 1.75$ & $2.58 \pm 1.66$ & $22.9 \pm 25.4$ & $1.11 \pm 0.55$ & $0.07 \pm 0.09$ \\
\hline $\mathrm{ZH}$ & $22.0 \pm 25.5$ & $2.47 \pm 1.24$ & $5.42 \pm 1.28$ & $44.9 \pm 24.9$ & $1.46 \pm 0.32$ & $0.07 \pm 0.03$ \\
\hline DL & $4.95 \pm 4.86$ & $1.75 \pm 0.94$ & $3.30 \pm 1.69$ & $22.3 \pm 11.9$ & $1.26 \pm 0.52$ & $0.08 \pm 0.04$ \\
\hline $\mathrm{XC}$ & $7.42 \pm 15.1$ & $1.02 \pm 0.60$ & $2.29 \pm 1.01$ & $27.8 \pm 22.8$ & $0.72 \pm 0.58$ & $0.19 \pm 0.10$ \\
\hline LT & $32.9 \pm 63.3$ & $1.47 \pm 1.59$ & $2.86 \pm 2.08$ & $46.7 \pm 39.5$ & $0.72 \pm 1.02$ & $0.18 \pm 0.13$ \\
\hline YK & $1.98 \pm 1.73$ & $0.95 \pm 1.59$ & $2.36 \pm 1.73$ & $13.4 \pm 10.2$ & $0.55 \pm 0.39$ & $0.10 \pm 0.08$ \\
\hline DY & $6.87 \pm 12.7$ & $0.72 \pm 0.45$ & $4.63 \pm 4.91$ & $79.5 \pm 128$ & $0.78 \pm 0.82$ & $0.17 \pm 0.15$ \\
\hline $\mathrm{TJ}$ & $9.99 \pm 14.8$ & $1.78 \pm 1.68$ & $3.93 \pm 2.30$ & $48.7 \pm 53.4$ & $1.19 \pm 0.84$ & $0.23 \pm 0.21$ \\
\hline LK & $67.7 \pm 94.0$ & $1.32 \pm 1.39$ & $8.38 \pm 8.99$ & $67.6 \pm 48.7$ & $1.51 \pm 1.17$ & $0.35 \pm 0.28$ \\
\hline YT & $19.3 \pm 27.6$ & $1.63 \pm 0.84$ & $5.01 \pm 3.62$ & $1606 \pm 1206$ & $0.59 \pm 0.36$ & $0.33 \pm 0.24$ \\
\hline $\mathrm{BH}$ & $56.3 \pm 57.8$ & $2.43 \pm 2.45$ & $14.6 \pm 18.2$ & $116 \pm 81.8$ & $2.10 \pm 1.68$ & $0.40 \pm 0.30$ \\
\hline $\mathrm{RC}$ & $8.15 \pm 10.1$ & $2.72 \pm 1.11$ & $3.10 \pm 1.08$ & $99.4 \pm 137$ & $0.51 \pm 0.19$ & $0.15 \pm 0.08$ \\
\hline BS & $23.5 \pm 24.1$ & $1.43 \pm 0.52$ & $5.29 \pm 3.97$ & $52.7 \pm 31.8$ & $1.10 \pm 0.49$ & $0.21 \pm 0.11$ \\
\hline NYS & $19.8 \pm 17.5$ & $2.40 \pm 0.60$ & $5.67 \pm 4.13$ & $319 \pm 577$ & $1.17 \pm 0.54$ & $0.18 \pm 0.14$ \\
\hline Average & $20.5 \pm 20.5$ & $1.81 \pm 0.77$ & $4.87 \pm 3.37$ & $183 \pm 430$ & $1.04 \pm 0.47$ & $0.19 \pm 0.11$ \\
\hline
\end{tabular}

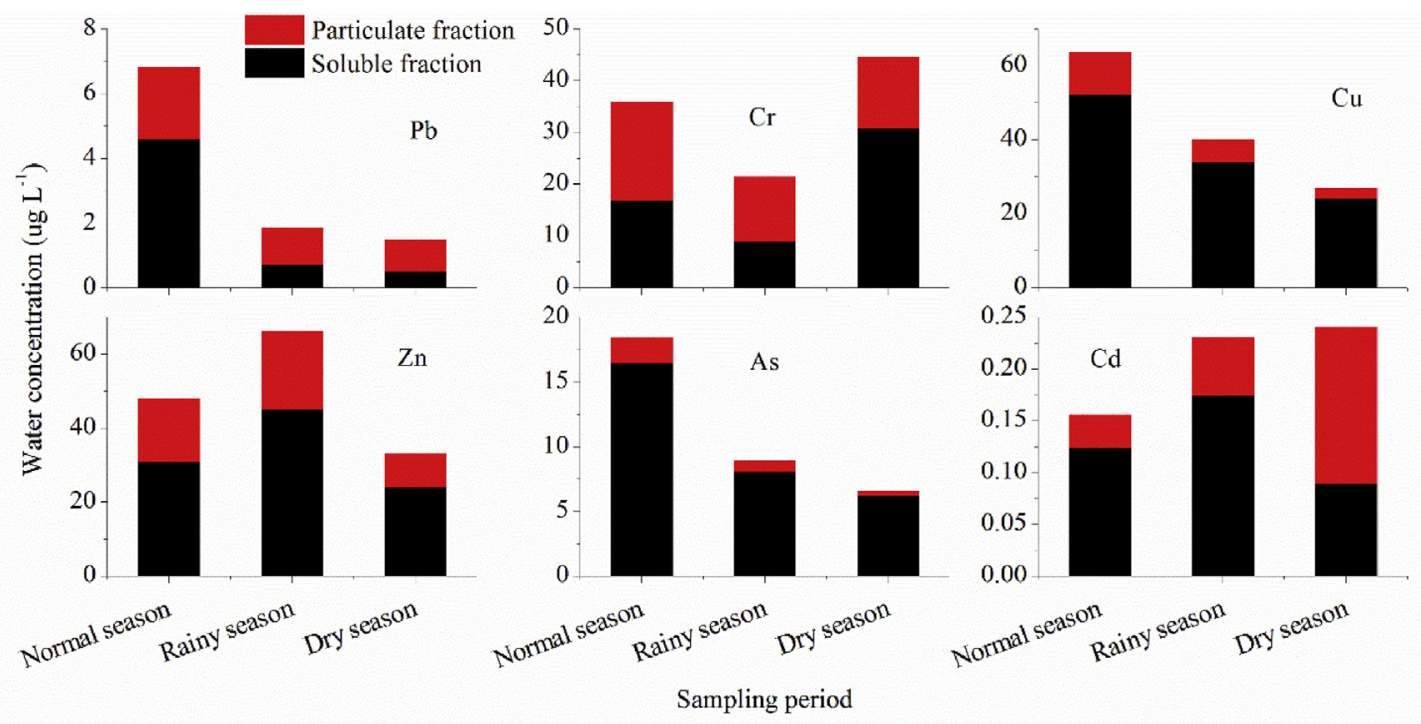

Fig. 2. Soluble and particulate concentrations of HMA in water averaged over 37 rivers around the BS and NYS

SI. The mean \pm standard deviations of total concentrations were $49.0 \pm 85.8 \mu \mathrm{g} \mathrm{L}^{-1} \quad$ for $\mathrm{Zn}, \quad 43.8 \pm 73.2 \mu \mathrm{g} \mathrm{L}^{-1}$ for $\mathrm{Cu}$, $33.9 \pm 42.8 \mu \mathrm{g} \mathrm{L}^{-1}$ for $\mathrm{Cr}, 11.3 \pm 16.7 \mu \mathrm{g} \mathrm{L}^{-1}$ for As, $3.42 \pm 4.79 \mu \mathrm{g} \mathrm{L}^{-1}$ for $\mathrm{Pb}$, and $0.21 \pm 0.39 \mu \mathrm{g} \mathrm{L}{ }^{-1}$ for $\mathrm{Cd}$. The highest and lowest HMA water concentrations in these rivers were $\mathrm{Zn}$ and $\mathrm{Cd}$, respectively, which agreed with their respective deposition rates as described in Section 3.1. A significant difference occurred between the Pb concentration in rivers and its atmospheric deposition rate, illustrated by the second highest $\mathrm{Pb}$ deposition and second lowest water concentration in the rivers among the six metals around the BS and NYS.

The highest concentrations of $\mathrm{Pb}, \mathrm{Cu}$ and As were discerned in the normal season, peak concentrations of $\mathrm{Cr}$ and $\mathrm{Cd}$ were identified in the dry season, and $\mathrm{Zn}$ concentrations reached a maximum in the rainy season. The difference in the temporal distribution among the sampled metals depends on usage and chemicalphysical properties of each element (IAEA, 2004; Xu et al., 2013a). The Student's test showed that, at a 5\% significance level, $\mathrm{Pb}$ and $\mathrm{As}$ concentrations in the normal season were significantly higher than those in other seasons, $\mathrm{Cr}$ concentrations in the dry and normal seasons were significantly higher than that in the rainy season, and $\mathrm{Cu}$ concentration in the normal season was significantly higher than that in the dry season (see Table S15 of SI). As also shown in
Table 14 of SI, the soluble concentrations of $\mathrm{Cu}, \mathrm{Zn}, \mathrm{As}$, and $\mathrm{Cd}$ accounted for major fractions of their respective total concentrations. The largest soluble fractions of these metals were $80.7 \pm 19.5 \%$ for $\mathrm{Cu}, 61.1 \pm 23.6 \%$ for $\mathrm{Zn}, 86.5 \pm 16.4 \%$ for As, and $55.2 \pm 30.1 \%$ for $\mathrm{Cd}$. However, the soluble fractions of $\mathrm{Pb}$ and $\mathrm{Cr}$ $(38.6 \pm 29.1 \%$ and $47.8 \pm 28.6 \%$, respectively) were lower than their particulate portions. The highest particulate HMA concentrations exhibited no significant changes in the three seasons except for the significantly high Cd concentration in the dry season compared with the other two seasons (see Table S15 of SI). Other significant variations in the soluble concentrations of the sampled HMA are shown in Table S15 of SI.

Large standard deviations in the HMA concentrations in the riverine waters (see Table S14 of SI) indicated their significant spatial differences. These rivers can be classified into four groups according to their discharge into the three bays of the BS and NYS. These are the Liaodong Bay, Bohai Bay, Laizhou Bay, and NYS as shown in Table S16 of SI. According to the classification, the rivers around the Laizhou Bay suffered from the most serious contamination by $\mathrm{Cu}, \mathrm{Zn}$, As, and $\mathrm{Cd}$, followed by $\mathrm{Pb}$ and $\mathrm{Cr}$. The mean $\mathrm{Pb}$ concentrations in the rivers entering the Bohai Bay were the highest, followed by other elements of HMA. These rivers provided major pathways that delivered HMA to these two bays. 
The mean HMA concentrations in the riverine water were compared with those in the seawater of the BS and NYS as shown in Table S17 of SI. In general, the HMA concentrations in the rivers were remarkably higher than those in seawater of the BS and NYS. The HMA levels followed a sequence of $\mathrm{Zn}>\mathrm{Cu}>\mathrm{Cr}>\mathrm{As}>\mathrm{Pb}>\mathrm{Cd}$. The HMA concentrations in the 37 rivers were also assessed in terms of third grade water quality in the Chinese Environmental Quality Standards for Surface Water (GB3838-2002) (see Table S18 of SI). Water quality standards for each element are often applied in water quality assessment of central drinking water resources, wintering grounds and migration channels of fish and shrimp, mariculture waters of fish, shrimp, shellfish, and algae, and bathing areas. As shown in Table S14 and Table S18 of SI, the mean concentrations of sampled HMA in the river waters were well below their respective standards except for $\mathrm{Cr}$ and As (Table S18 of SI). For these two elements, $\mathrm{Cr}$ exceeded its standard by $27 \%$ in the normal season, $8 \%$ in the rainy season, and $25 \%$ in the dry season, and As exceeded its standard by $16 \%, 3 \%$, and $0 \%$ for these three seasons, respectively. The estuaries of most of the rivers with elevated levels of $\mathrm{Cr}$ and As were located in the Bohai Bay as shown in Table S18 of SI.

For individual water samples, dominant excesses were found for $\mathrm{Cr}$ and As as shown in Table S18 of SI. The excessive percentages in normal, rainy and dry seasons were $27 \%, 8 \%$, and $25 \%$ for $\mathrm{Cr}$ and $16 \%$, $3 \%$, and $0 \%$ for As, respectively. The estuaries of most of the rivers with elevated levels of $\mathrm{Cr}$ and As were located in the Bohai Bay as shown in Table S18 of SI.

\subsection{Fluxes of HMA to the Bohai and North Yellow seas}

To be consistent, the deposition fluxes and river discharges of HMA into the BS and NYS were calculated for the year 2015, and the results are summarized in Fig. 3 and Table S19 of SI. For the entire BS and NYS, the HMA fluxes via river inflows were 3-20 times higher than the atmospheric deposition fluxes except for $\mathrm{Pb}$, thereby suggesting that the dominant input pathway of HMA was by river discharges. However, in the BS's subareas, the atmospheric deposition fluxes of $\mathrm{Zn}$ to the Liaodong Bay and $\mathrm{Cd}$ to the Bohai Bay were higher than those via river discharge. This finding verified a hypothesis that $\mathrm{Pb}$ in the Bohai Bay originated primarily from atmospheric deposition after 2001 (Meng et al., 2008). It also demonstrated that the contamination of $\mathrm{Pb}, \mathrm{Cd}$, and $\mathrm{Zn}$ in the Liaodong Bay was largely attributed to atmospheric deposition (Zheng et al., 2008), and that most HMA in the Laizhou Bay originated from the Yellow River (Bi et al., 2014; Lü et al., 2015; Zhang et al., 2017a).

The total $\mathrm{Pb}$ inputs were the largest in the NYS, followed by the Central BS, Liaodong Bay, Bohai Bay, and Laizhou Bay, which, to a large extent, was related to the area of these waters (see Table S3 of SI). This is because atmospheric deposition was the dominant input pathway of $\mathrm{Pb}$, and the deposition flux depends on the receiving area. The rank of total annual inputs of $\mathrm{Cr}, \mathrm{Cu}, \mathrm{Zn}$, and As in the sea areas exhibited the same trend of the NYS $>$ Laizhou Bay $>$ Bohai Bay $>$ Liaodong Bay $>$ Central Bohai because of the magnitude of their input mass via river discharge. The largest inputs of $\mathrm{Cd}$ were in the Laizhou Bay because of the major discharges of trace metals via the Yellow River (Lü et al., 2015; Zhang et al., 2017a). In fact, the Yellow River is a leading channel for all HMA entering the Laizhou Bay. The river contributed $87.3 \pm 11.4 \%, 83.3 \pm 9.70 \%, 77.4 \pm 13.2 \%$, $91.1 \pm 12.8 \%, 69.8 \pm 10.9 \%$, and $91.9 \pm 10.8 \%$ to the total river inputs of $\mathrm{Pb}, \mathrm{Cr}, \mathrm{Cu}, \mathrm{Zn}, \mathrm{As}$, and $\mathrm{Cd}$ into the Laizhou Bay, respectively (see Table S20 of SI). Likewise, the Yalu River contributed over 70\% of total HMA river inputs into the NYS as shown in Table S20 of SI (Li et al., 2014). Such a dominant contribution of a river discharge was not observed in the Liaodong Bay or Bohai Bay. The largest contributions of HMA discharges from rivers connected with the two bays were less than $50 \%$ as shown in Table S20 of SI.

The total inputs of HMA through atmospheric deposition and riverine inflow to the BS's four subareas and the NYS can be converted into concentrations (see Table S21 of SI) by dividing fluxes by water volumes in each sea area. Concentration levels of HMA in seawater were widely used to assess their ecological risks using the method of risk quotient (RQ) (Wang et al., 2010). RQ value of HMA can be calculated by comparing their measured or predicted concentrations with appropriate threshold values. This study used the converted HMA concentrations as their predicted levels and the Grade-I seawater quality standards of China (GB 3097-1997, China, see Table S21 of SI) (Peng, 2015) as threshold value to assess the health risk subject to estimated RQ values (see Table S22 of SI). The criteria are set as precautionary measure for protecting the fishing water and endangered marine species to avoid chronic toxicity of chemicals like HMA (Wang et al., 2010). The RQ values of Pb, $\mathrm{Cu}$, and

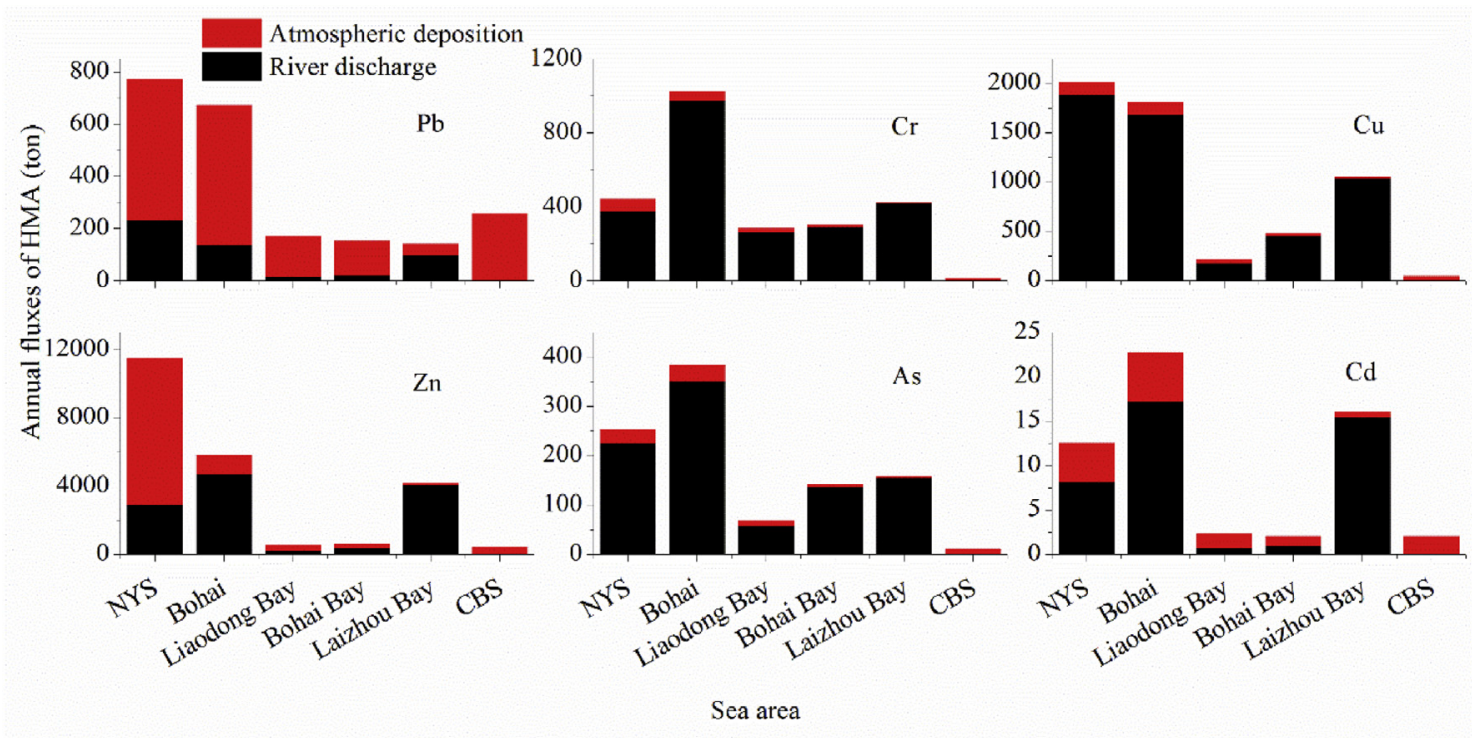

Fig. 3. Annual fluxes of HMA contributed by atmospheric deposition and river discharge into the BS and NYS. 
$\mathrm{Zn}$ in Laizhou Bay and the RQ value of $\mathrm{Pb}$ in Bohai Bay were greater than 1 , indicating that annual metal input posed a considerable threat to the marine ecosystem of the two bays if these HMA entered into in seawater.

Compared with HMA concentrations measured in the BS and NYS (see Table S17 of SI), the annual HMA fluxes only contributed $1-10 \%$ to measured pollution levels in the Liaodong Bay. In the Bohai Bay, the converted concentrations of $\mathrm{Cr}, \mathrm{Cu}$, and As were higher than their respective monitored concentrations, although the fluxes posed a minor contribution to $\mathrm{Pb}, \mathrm{Zn}$, and $\mathrm{Cd}$. Specifically, the HMA fluxes contributed to higher concentrations than the contamination levels in the Laizhou Bay. This suggests that the HMA entering the BS were not only presence in the waterbody, but were also more likely to migrate to the sediments and to the outer seas (such as the NYS). The HMA outflows of HMA and/or the buried HMA were the most significant in the Laizhou Bay, followed by the Bohai Bay and Liaodong Bay.

\subsection{Source-sink relationship of HMA in the Bohai and North Yellow seas}

Table S23of SI lists the HMA burdens in seawater and sediments, as well as the exchange capacity of HMA among the BS's four subareas and the NYS after 1-year of water exchange. Except for the Central BS, the HMA fluxes via atmospheric deposition and river discharge (see Table S19 of SI) into each sea area were larger than the corresponding exchange capacity (see Table S23 of SI), thereby suggesting the dominant pathway of the terrestrial inputs. For instance, the $\mathrm{Pb}$ inputs through atmospheric deposition and river inflow into the Liaodong Bay were $155 \pm 220$ ton $\mathrm{yr}^{-1}$ and $17.0 \pm 22.4$ ton $\mathrm{yr}^{-1}$, respectively. Among the total inputs, 114 tons remained in the Liaodong Bay, and 7.24 tons, 42.1 tons and 7.91 tons were transported into the Bohai Bay, Central BS and NYS, respectively, after the 1-year water exchange. In the meantime, 7.46 tons, 2.42 tons, 18.7 tons, and 43.9 tons of $\mathrm{Pb}$ were entered into the Liaodong Bay from the Bohai Bay, Laizhou Bay, Central BS and NYS, respectively. The inflows by the water exchange were less than the total input via atmospheric deposition and river discharge.

The HMA loads into the Central BS were mainly through water exchanges among the sea areas, rather than through direct terrestrial inputs. For instance, the inputs of $\mathrm{Cr}, \mathrm{Cu}, \mathrm{Zn}, \mathrm{As}$, and $\mathrm{Cd}$ from the Laizhou Bay were 183 ton $\mathrm{yr}^{-1}, 535$ ton $\mathrm{yr}^{-1}, 1720$ ton $\mathrm{yr}^{-1}$, 67.0 ton $\mathrm{yr}^{-1}$ and 7.54 ton $\mathrm{yr}^{-1}$, respectively, which were clearly higher than the deposition input (see Table S19 and Table S23 of SI). The second largest contributor to the HMA contamination in the Central BS was the Bohai Bay; there was larger HMA inflow from this bay than from the total terrestrial inputs, which was in agreement with the source-sink relationship between these two sea areas. The majority of HMA outflows from the Central BS entered the NYS, and the second largest outflow entered the Liaodong Bay. The HMA exports from the NYS follow two major pathways. One pathway was to the Central BS, and the other extended to the Liaodong Bay. These inflows and outflows indicated that the Central BS and NYS were the transition region for most HMA through water exchange.

The total HMA burdens in the BS's four subareas and in the NYS were significantly different (see Table S23 of SI). The fractions of HMA to the total burdens in the waterbody were $86.6 \pm 4.55 \%$ in the Liaodong Bay, $60.5 \pm 10.5 \%$ in the Bohai Bay, $20.9 \pm 9.05 \%$ in the Laizhou Bay, $95.1 \pm 2.06 \%$ in the Central BS, and $94.3 \pm 1.93 \%$ in the NYS. The lowest HMA fraction in the Laizhou Bay could be attributed to its high sedimentation rate and SPM concentrations, which originated largely from the Yellow River (Bi et al., 2014; Tang et al., 2010). The ranking of HMA sediment burdens in each sea area was similar to that of HMA concentrations in the sediments of the BS and NYS that were determined in previous studies (see Table S24 of SI).

According to the mass balance of HMA, a sea area could be a source of a pollutant for other sea areas if the total terrestrial inputs of the pollutant are larger than its total burdens (including seawater and sediments) in the sea area. On the other hand, the sea area can also be considered a sink area if the total inputs are less than the total burdens. To explore which role each sea area played in the HMA mass balance, Table S25 of SI lists the percentages of water volume and HMA in each sea area compared with the total sea area and total HMA. Table S26 of SI shows the total inputs and total burdens of HMA, as well as their budget after 1-year of water exchange within the BS's four subareas and the NYS. The budget was defined as the difference between the total inputs and total burdens of HMA. As shown, the Liaodong Bay was a sink of $\mathrm{Pb}, \mathrm{Cu}$, $\mathrm{Zn}$, As, and $\mathrm{Cd}$ because their inputs were smaller than the total burdens (see Table S26 of SI). This indicated that most HMA in the bay were attributed not only to the terrestrial inputs, but also to the seawater exchange among the several sea areas. The terrestrial input of $\mathrm{Cr}$ was the highest among the six HMA in the Liaodong Bay, and accounted for $19.3 \%$ of the total inputs in the BS and NYS (see Table S25 of SI). The contribution percentage was higher than the percentage (14.6\%) of water volume in the Liaodong Bay to the total volume (see Table S25 of SI). As a result, the Liaodong Bay was a $\mathrm{Cr}$ source instead of a sink. Likewise, the Bohai Bay and Laizhou Bay were mainly sources of HMA because their contribution percentages were greater than the water volume percentages $(2.32 \%$ and $1.32 \%$ for the Bohai Bay and Laizhou Bay, respectively, see Table S25 of SI). In contrast, the total burdens of HMA in the Central BS were larger than the total inputs, thereby indicating that this sea area was a sink of HMA (see Table S20 of SI), which could be attributed to the relatively weak terrestrial inputs of HMA. The NYS could be regarded as a weak source of HMA, except for $\mathrm{Pb}$, because the inputs were less than the total burdens (see Table S26 of SI). To further explore the source-sink roles of each sea area, the annual budgets of HMA after 1-, 10-, and 100- year exchanges are displayed in Fig. 4. The Liaodong Bay as a sink of HMA can be more clearly seen from the growing negative differences within these time periods, thereby suggesting that the mitigation of water pollution in the Liaodong Bay becomes much more difficult due to its historical contamination by HMA. In general, the Laizhou Bay and Bohai Bay acted as sources of HMA, as shown by the positive differences between the total inputs and total burdens. Both positive and negative differences from the three simulations further indicated that the Central BS and NYS underwent sink to source reversals for most HMA. The source-sink relationship of HMA in the BS and NYS indicates that systematic cooperation of administrative districts near the BS and NYS to mitigate HMA input from rivers and atmosphere is the effective strategies to improve and restore their ecosystem, and Liaodong Bay should be pay to a specific attention (Song et al., 2017).

\section{Conclusions}

The observations and simulations based on comprehensive data on the atmospheric deposition and river water samples around the BS and NYS allowed us to draw the following major conclusions. (1) The fractions of the dissolved HMA in the atmospheric deposition samples were higher than those in the river water samples. The spatiotemporal variation in HMA deposition rates was insignificant, whereas water concentrations of the HMA had significant seasonal variation. (2) For the entire BS and NYS, atmospheric deposition dominated the input of $\mathrm{Pb}$, and river discharge was largely responsible for the input of the other $\operatorname{HMA}(\mathrm{Cr}, \mathrm{Cu}, \mathrm{Zn}, \mathrm{As}$, and $\mathrm{Cd})$. (3) The terrestrial input of HMA into the BS and NYS indicated that 


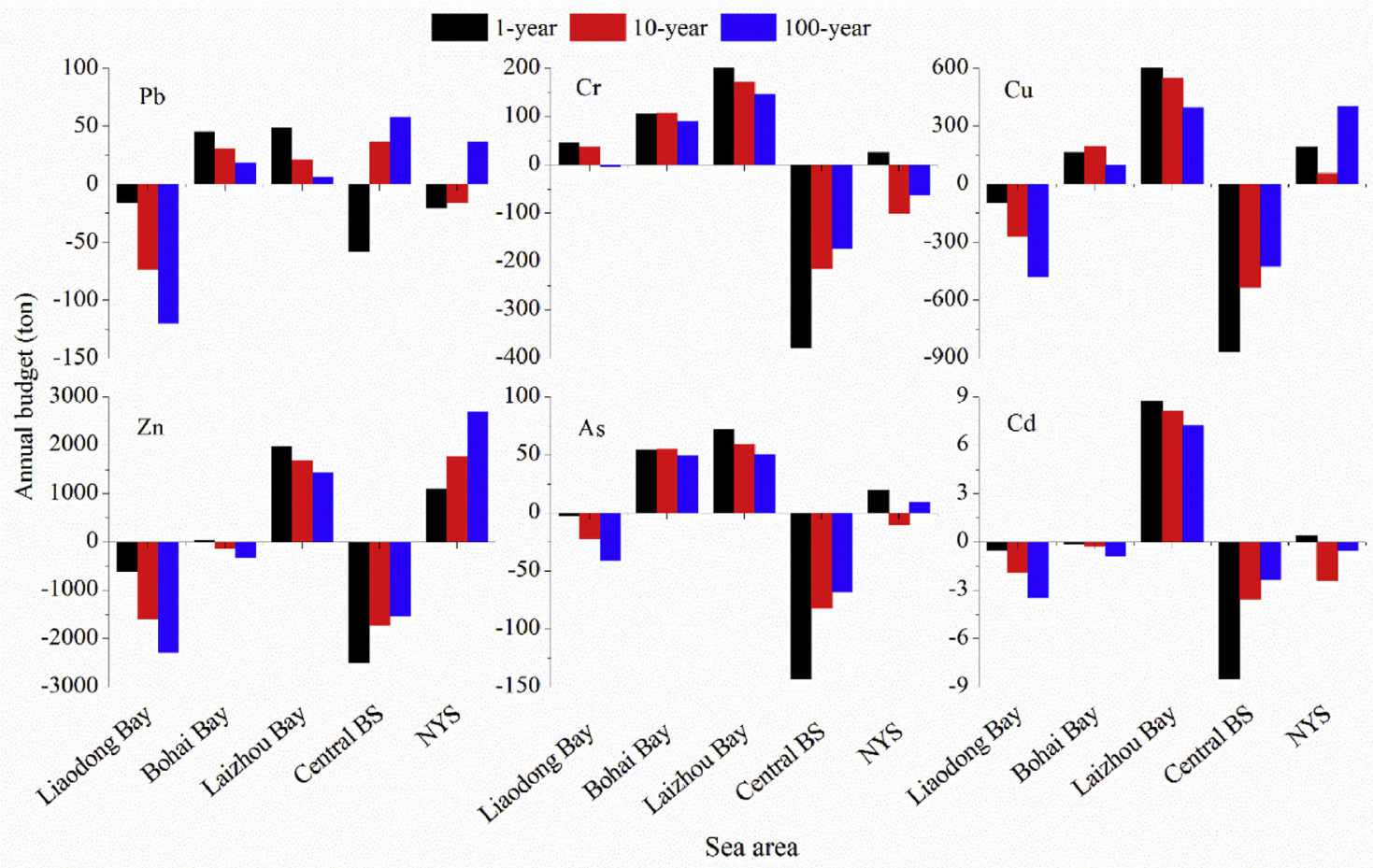

Fig. 4. Annual mean budgets of HMA in the BS's four subareas and the NYS after 1-, 10-, and 100-year of water exchange.

the Laizhou Bay and Bohai Bay were sources, the Liaodong Bay was a sink, and that the Central BS and NYS were a transition region through water exchange.

\section{Notes}

The authors declare no competing financial interest.

\section{Acknowledgment}

This work was financially supported by the Strategic Priority Research Program of the CAS (XDA11020402) and the Natural Scientific Foundation of China (41471413).

\section{Appendix A. Supplementary data}

Supplementary data related to this article can be found at https://doi.org/10.1016/j.envpol.2018.08.011.

\section{References}

Bi, Naishuang, Yang, Zuosheng, Wang, Houjie, Xu, Congliang, Guo, Zhigang, 2014. Impact of artificial water and sediment discharge regulation in the Huanghe (Yellow River) on the transport of particulate heavy metals to the sea. CATENA $121,232-240$.

Duan, Jingchun, Tan, Jihua, 2013. Atmospheric heavy metals and Arsenic in China: situation, sources and control policies. Atmos. Environ. 74 (0), 93-101.

Duan, Xiaoyong, Li, Yanxia, 2017. Distributions and sources of heavy metals in sediments of the Bohai Sea, China: a review. Environ. Sci. Pollut. Res. 24 (32), 24753-24764.

Fang, Yin, Chen, Yingjun, Tian, Chongguo, Tian, Lin, Hu, Limin, Huang, Guopei, Tang, Jianhui, Li, Jun, Zhang, Gan, 2015. Flux and budget of BC in the continental shelf seas adjacent to Chinese high BC emission source regions. Global Biogeochem. Cycles 29 (7), 957-972.

Gao, Xuelu, Zhou, Fengxia, Arthur, Chen Chen-Tung, 2014. Pollution status of the Bohai Sea: an overview of the environmental quality assessment related trace metals. Environ. Int. 62 (0), 12-30.

Guo, Lanlan, Lyu, Yanli, Yang, Yanyan, 2017. Concentrations and chemical forms of heavy metals in the bulk atmospheric deposition of Beijing, China. Environ. Sci. Pollut. Res. 24 (35), 27356-27365.
Hu, Ningjing, Liu, Jihua, Huang, Peng, Yan, Shijuan, Shi, Xuefa, Ma, Deyi, 2017 Sources, geochemical speciation, and risk assessment of metals in coastal sediments: a case study in the Bohai Sea, China. Environ. Earth Sci. 76 (8), 309.

IAEA, 2004. Sediment Distribution Coefficients and Concentration Factors for Biota in the Marine Environment (Technical reports series, no. 422). International Atomic Energy Agency, Vienna, pp. 8-25.

Lü, Dongwei, Zheng, Bing, Yan, Fang, Shen, Ge, Liu, Huijun, 2015. Distribution and pollution assessment of trace metals in seawater and sediment in Laizhou Bay. Chin. J. Oceanol. Limnol. 33 (4), 1053-1061.

Li, Jianchao, Li, Guangxue, Xu, Jishang, Dong, Ping, Qiao, Lulu, Liu, Shidong, Sun, Pingkuo, Fan, Zhisong, 2016. Seasonal evolution of the Yellow Sea cold water mass and its interactions with ambient hydrodynamic system. J. Geophys. Res.: Oceans 121 (9), 6779-6792.

Li, Zi-Ye, Liu, Dong-Sheng, Long, Hai-Yan, 2014. Living and dead benthic foraminifera assemblages in the Bohai and northern Yellow Seas: seasonal distributions and paleoenvironmental implications. Quat. Int. 349, 113-126.

Liu, Jian, Saito, Yoshiki, Kong, Xianghuai, Wang, Hong, Zhao, Ling, 2009. Geochemical characteristics of sediment as indicators of post-glacial environmental changes off the Shandong Peninsula in the Yellow Sea. Continent. Shelf Res. 29 (7), 846-855.

Liu, Wenchuan, Hu, Limin, Tian, Lin, Li, Yuanyuan, Guo, Zhigang, 2017. Distribution and mass inventory of mercury in sediment from the Yangtze River estuarineinner shelf of the East China Sea. Continent. Shelf Res. 132, 29-37.

Liu, Yansui, Hu, Zhichao, Li, Yuheng, 2014. Process and cause of urban-rural development transformation in the Bohai Rim Region, China. J. Geogr. Sci. 24 (6), 1147-1160.

Meng, Jing, Hong, Seongjin, Wang, Tieyu, Li, Qifeng, Yoon, Seo Joon, Lu, Yonglong, Giesy John, P., Khim, Jong Seong, 2017. Traditional and new POPs in environments along the Bohai and Yellow seas: an overview of China and South Korea. Chemosphere 169, 503-515.

Meng, Wei, Qin, Yanwen, Zheng, Binghui, Zhang, Lei, 2008. Heavy metal pollution in Tianjin Bohai bay, China. J. Environ. Sci. 20 (7), 814-819.

Pan, Ke, Wang, Wen-Xiong, 2012. Trace metal contamination in estuarine and coastal environments in China. Sci. Total Environ. 421-422, 3-16.

Pan, Y.P., Wang, Y.S., 2015. Atmospheric wet and dry deposition of trace elements at 10 sites in Northern China. Atmos. Chem. Phys. 15 (2), 951-972.

Peng, Shitao, 2015. The nutrient, total petroleum hydrocarbon and heavy metal contents in the seawater of Bohai Bay, China: temporal-spatial variations, sources, pollution statuses, and ecological risks. Mar. Pollut. Bull. 95 (1), 445-451.

Sharma, Rajesh Kumar, Agrawal, Madhoolika, Marshall Fiona, M., 2008. Atmospheric deposition of heavy metals (Cu, $\mathrm{Zn}, \mathrm{Cd}$ and $\mathrm{Pb}$ ) in Varanasi City, India. Environ. Monit. Assess. 142 (1), 269-278.

SOA(State-Oceanic-Administration-of-China), Dec. 04, 2017. Marine environmental quality bulletin of China in 2016. http://www.soa.gov.cn/zwgk/hygb/ zghyhjzlgb/201712/t20171204_59423.html. 
Song, Nan-Qi, Wang, Nuo, Lin, Wan-Ni, Wu, Nuan, 2017. Use of pollution risk and ecosystem service values in marine management strategies in Bohai Sea, China. Hum. Ecol. Risk Assess. 23 (5), 1041-1055.

Su, Shenshen, Li, Bengang, Cui, Siyu, Shu, Tao, 2011. Sulfur dioxide emissions from combustion in China: from 1990 to 2007. Environ. Sci. Technol. 45 (19), 8403-8410.

Sun, Jian, Tao, Jianhua, 2006. Relation matrix of water exchange for sea bays and its application. China Ocean Eng. 20 (4), 529-544.

Tang, Aikun, Liu, Ruhai, Min, Ling, Xu, Liaoqi, Wang, Jinyu, 2010. Distribution characteristics and controlling factors of soluble heavy metals in the Yellow River Estuary and adjacent sea. Procedia Environ. Sci. 2, 1193-1198.

Wang, Chang-you, Wang, Xiu-lin, 2007. Spatial distribution of dissolved Pb, Hg, Cd, Cu and as in the Bohai Sea. J. Environ. Sci. 19 (9), 1061-1066.

Wang, Ji-Zhong, Guan, Yu-Feng, Ni, Hong-Gang, Luo, Xian-Lin, Zeng Eddy, Y., 2007. Polycyclic aromatic hydrocarbons in riverine runoff of the Pearl River Delta (China): Concentrations, fluxes, and fate. Environ. Sci. Technol. 41 (16), 5614-5619.

Wang, Jing, Shang, Chen, Xia, Tao, 2010. Environmental risk assessment of heavy metals in Bohai Sea, North China. Procedia Environ. Sci. 2, 1632-1642.

Wang, Runmei, Tang, Jianhui, Xie, Zhiyong, Mi, Wenying, Chen, Yingjun, Hendrik, Wolschke, Tian, Chongguo, Pan, Xiaohui, Luo, Yongming, Ralf, Ebinghaus, 2015. Occurrence and spatial distribution of organophosphate ester flame retardants and plasticizers in 40 rivers draining into the Bohai Sea, north China. Environ. Pollut. 198 (0), 172-178.

Wang, Yan, Ru-hai, Liu, Zhang, Yu-qing, Xue-qing, Cui, Tang, Ai-kun, Zhang, Longjun, 2016. Transport of heavy metals in the Huanghe River estuary, China. Environ. Earth Sci. 75 (4), 288.

Wong, C.S.C., Li, X.D., Zhang, G., Qi, S.H., Peng, X.Z., 2003. Atmospheric deposition of heavy metals in the Pearl River Delta, China. Atmos. Environ. 37 (6), 767-776.

Wu, Ting, Hou, Xiyong, Chen, Qing, 2016. Coastal economic vulnerability to sea leve rise of Bohai Rim in China. Nat. Hazards 80 (2), 1231-1241.

Xing, Jianwei, Song, Jinming, Yuan, Huamao, Wang, Qidong, Li, Xuegang, Ning, Li, Duan, Liqin, Qu, Baoxiao, 2017. Atmospheric wet deposition of dissolved trace elements to Jiaozhou Bay, North China: fluxes, sources and potential effects on aquatic environments. Chemosphere 174, 428-436.

Xu, Li, Wang, Tieyu, Ni, Kun, Liu, Shijie, Wang, Pei, Xie, Shuangwei, Meng, Jing, Zheng, Xiaoqi, Lu, Yonglong, 2013a. Metals contamination along the watershed and estuarine areas of southern Bohai Sea, China. Mar. Pollut. Bull. 74 (1) 453-463.

Xu, Yue, Tian, Chongguo, Ma, Jianmin, Wang, Xiaoping, Li, Jun, Tang, Jianhui, Chen, Yingjun, Qin, Wei, Zhang, Gan, 2013b. Assessing cancer risk in China from $\gamma$-Hexachlorocyclohexane emitted from Chinese and Indian sources. Environ.
Sci. Technol. 47 (13), 7242-7249.

Yang, Xiaolong, Yuan, Xiutang, Zhang, Anguo, Mao, Yuze, Oiang, Li, Zong, Humin, Wang, Lijun, Li, Xiaodong, 2015. Spatial distribution and sources of heavy metals and petroleum hydrocarbon in the sand flats of Shuangtaizi Estuary, Bohai Sea of China. Mar. Pollut. Bull. 95 (1), 503-512.

Zhang, Fan, Chen, Yingjun, Oi, Chen, Feng, Yanli, shang, Yu, Yang, Xin, Gao, Huiwang, Tian, Chongguo, Li, Jun, Zhang, Gan, Volker, Matthias, Xie, Zhiyong, 2018. Realworld emission factors of gaseous and particulate pollutants from marine fishing boats and their total emissions in China. Environ. Sci. Technol. 52 (8), 4910-4919.

Zhang, Jinfeng, Gao, Xuelu, 2015. Heavy metals in surface sediments of the intertidal Laizhou Bay, Bohai Sea, China: distributions, sources and contamination assessment. Mar. Pollut. Bull. 98 (1), 320-327.

Zhang, P., Song, J., Yuan, H., 2009. Persistent organic pollutant residues in the sediments and mollusks from the Bohai Sea coastal areas, North China: an overview. Environ. Int. 35 (3), 632-646.

Zhang, Pan, Hu, Rijun, Zhu, Longhai, Wang, Peng, Yin, Dongxiao, Zhang, Lianjie, 2017a. Distributions and contamination assessment of heavy metals in the surface sediments of western Laizhou Bay: implications for the sources and influencing factors. Mar. Pollut. Bull. 119 (1), 429-438.

Zhang, Yanling, Zhang, Shixiang, Zhu, Fengpeng, Wang, Aiguo, Dai, Huaxin, Cheng, Sen, Wang, Jianwei, Tang, Lina, 2017b. Atmospheric heavy metal deposition in agro-ecosystems in China. Environ. Sci. Pollut. Res. 25 (6), 5822-5831.

Zheng, Na, Wang, Qichao, Liang, Zhongzhu, Zheng, Dongmei, 2008. Characterization of heavy metal concentrations in the sediments of three freshwater rivers in Huludao City, Northeast China. Environ. Pollut. 154 (1), 135-142.

Zhu, Jianxing, Wang, Qiufeng, Yu, Haili, Li, Meiling, He, Nianpeng, 2016. Heavy metal deposition through rainfall in Chinese natural terrestrial ecosystems: evidences from national-scale network monitoring. Chemosphere 164, 128-133.

Zong, Zheng, Wang, Xiaoping, Tian, Chongguo, Chen, Yingjun, Fang, Yunting, Zhang, Fan, Cheng, Li, Sun, Jianzhong, Li, Jun, Zhang, Gan, 2017. First assessment of $\mathrm{NO}_{\mathrm{x}}$ sources at a regional background site in North China using isotopic analysis linked with modeling. Environ. Sci. Technol. 51 (11), 5923-5931.

Zong Zheng Wang Xiaoping, Tian, Chongouo, Chen, Yingjun, Fu, Shanfei, Ou, Lin, Ling, Ji, Li, Jun, Zhang, Gan, 2018. PMF and PSCF based source apportionment of $\mathrm{PM}_{2.5}$ at a regional background site in North China. Atmos. Res. 203, 207-215.

Zong Zheng, Wang Xiaoping, Tian, Chongguo, Chen, Yingiun, Qu, Lin, Ling, Ji, Guorui, Zhi, Li, Jun, Zhang, Gan, 2016. Source apportionment of $\mathrm{PM}_{2.5}$ at a regional background site in North China using PMF linked with radiocarbon analysis: insight into the contribution of biomass burning. Atmos. Chem. Phys. 16 (17), 11249-11265. 